\title{
Effect of Hydrodynamic Interactions on the Distribution of Adhering Brownian Particles
}

\author{
J. Bafaluy, ${ }^{(1),(2)}$ B. Senger, ${ }^{(1)}$, J.-C. Voegel, ${ }^{(1)}$ and P. Schaaf ${ }^{(3),(4)}$ \\ ${ }^{(1)}$ Institut National de la Sauté et de la Recherche Médicale CJF 92-04, Centre de Recherches Odontologiques, \\ Université Louis Pasteur, 1 Place de l'Hôpital, 67000 Strasbourg, France \\ ${ }^{(2)}$ Departament de Física, Universitat Autònoma de Barcelona, 08193 Bellaterra, Barcelona, Spain \\ ${ }^{(3)}$ Ecole Européenne des Hautes Etudes des Industries Chimiques de Strasbourg, \\ 1 rue Blaise Pascal, BP 296F, 67008 Strasbourg, France \\ ${ }^{(4)}$ Institut Charles Sadron, 6 rue Boussingault, 67083 Strasbourg, France
}

(Received 19 November 1992)

\begin{abstract}
Brownian dynamics simulations were used to study the adhesion of hard spheres on a solid surface by taking the hydrodynamic interactions into account. Special attention was paid to analyze the configuration of the assembly of adsorbed particles. These results were compared to configurations generated by the extensively studied random sequential adsorption (RSA) model. In our case the adsorption probability for a particle is almost uniform over the entire available surface. This surprising result shows that RSA provides a good approximation to generate adsorbed particle configurations.
\end{abstract}

PACS numbers: 68.10.Jy, 05.40.+j, 47.15.Gf

The adhesion of Brownian particles on solid surfaces has attracted much interest from both theoretical and experimental points of view during the last years. One of the most popular models used to describe this apparently simple process is the so-called random sequential adsorption (RSA) model [1], which takes excluded volume effects into account. However, it has serious limitations. In particular, it does not account for the transport of the particles to the adsorbing surface. This effect is introduced in the diffusion RSA model (DRSA) [2], where the adsorbing particle is allowed to diffuse in three-dimensional space subject to hard sphere interactions with previously adsorbed ones. The DRSA leads to an increased adsorption probability for an incoming sphere in the close vicinity of an already attached one as compared to RSA. The DRSA distribution of adsorbed particles is thus different at a given coverage from its RSA counterpart except near saturation (jamming limit) [3]. However, since the diffusion coefficient is taken constant, the DRSA seems to model a particle as moving in "dry water" [4]. This Letter is devoted to take hydrodynamic interactions into account and should thus represent a significant jump toward reality.

The effect of the hydrodynamic interaction is to increase the frictional force experienced by a particle when it approaches another one or a flat surface [5]. This kind of interaction between a sphere and a clean wall is well known, and its effect on the rate of adsorption has already been studied [6]. The main goal of this Letter is to investigate the influence of the hydrodynamic interactions between an adsorbing particle and (i) the already adsorbed ones, and (ii) the planar adsorbing surface, on the distribution of the particles on this surface. It will in particular be compared to RSA distributions in order to investigate the degree of accuracy of this simple and now well-known algorithm.
The Brownian motion of a spherical particle is completely described by the friction tensor, which is in general position dependent and nonisotropic. In particular, the normal component of the friction tensor diverges when the separation between the particle and any solid surface vanishes due to lubrication forces. As a consequence, contact of the particles with the adsorbing surface is impossible in the absence of a strong attractive force, like the van der Waals attraction. The latter becomes strong enough at small separations to overcome the lubrication effects. In general van der Waals, hard core, and electrostatic forces also act between the particles. However, in this study we will solely consider, besides the hydrodynamic interactions, the different hard core repulsions, and the van der Waals forces. For the latter we restrict ourselves to the forces acting between the moving sphere and the adsorbing plane. The sphereplane van der Waals force is derived from the corresponding potential, $U$, given by [7]

$$
U=-\frac{A_{H}}{6}\left[\frac{a}{s}+\frac{a}{s+2 a}+\ln \left(\frac{s}{s+2 a}\right)\right],
$$

where $s$ represents the shortest distance between the sphere of radius $a$ and the plane. The Hamaker constant $A_{H}$ corresponds to the interaction between the particle and the surface in the presence of the fluid. It is typically of the order of $10^{-20} \mathrm{~J}$.

The diffusion tensor $\mathbf{D}$ is related to the friction tensor $\mathbf{R}$ through the Einstein relation $\mathbf{D}=k T \mathbf{R}^{-1}$. Far from the surface, $\mathbf{D}$ becomes independent of the position of the sphere and is then given by the Stokes-Einstein relation $\mathbf{D}(\infty)=\mathbf{I} k T / 6 \pi \eta a, \eta$ being the viscosity of the fluid and I the unit tensor. In general, the position dependence of the diffusion tensor may be derived as follows. Using the linear dependence of the force $\mathbf{F}$ and torque $\mathbf{L}$ with the translational and angular velocities, $\mathbf{u}$ and $\boldsymbol{\Omega}$, respec- 
tively, one has according to Ref. [8] $\mathbf{F}=\mathbf{A} \cdot \mathbf{u}+\tilde{\mathbf{B}} \cdot \boldsymbol{\Omega}$, $\mathbf{L}=\mathbf{B} \cdot \mathbf{u}+\mathbf{C} \cdot \boldsymbol{\Omega}$, where the second rank tensors $\mathbf{A}, \mathbf{B}$, and $\mathbf{C}$ are components of the resistance matrix. $\tilde{\mathbf{B}}$ represents the transpose of matrix $\mathbf{B}$. For an isolated sphere there is no coupling between the torque and the translational motion $(\mathbf{B}=\mathbf{0})$. However, in the presence of boundaries, e.g., a sphere near a surface, the hydrodynamic interaction produces such a coupling. After elimination of the angular velocity in the previous linear equations, one finds $\mathbf{F}-\mathbf{B} \cdot \mathbf{C}^{-1} \cdot \mathbf{L}=\left(\mathbf{A}-\tilde{\mathbf{B}} \cdot \mathbf{C}^{-1} \cdot \mathbf{B}\right) \cdot \mathbf{u}$. This shows that the effective force acting on the particle, $\mathbf{F}_{\text {eff }}=\mathbf{F}-\mathbf{B} \cdot \mathbf{C}^{-1} \cdot \mathbf{L}$, is linearly related to the velocity through the effective friction tensor $\mathbf{R}_{\text {eff }}=\mathbf{A}-\tilde{\mathbf{B}} \cdot \mathbf{C}^{-1} \cdot \mathbf{B}$. This is the friction tensor that must be used in the Einstein relation to obtain the diffusion tensor needed in the Smoluchowski equation which governs the diffusion of the particles.

The lubrication forces depend on the local flow of the fluid in the small regions between the moving particle and (i) the plane and (ii) the adsorbed spheres. Since these regions are well disconnected, one can assume as a first approximation that they contribute additively to the resulting force, and thus also additively to the effective friction tensor [9]. The contributions from the spheresphere interactions to the friction tensor were calculated by using the analytical results given in Ref. [8]. The sphere-plane contributions were computed according to Refs. $[10,11]$.

The hydrodynamic interaction increases the friction experienced by a particle that approaches a surface. This increase is larger for the motion normal to the surface than for the parallel one. Therefore, the lateral diffusion of the particle is enhanced compared to the diffusion in the direction perpendicular to the surface. A randomization of the adsorption location along the surface is then expected, rendering the distribution of the adsorbed particles to look more like its RSA counterpart.

For low to intermediate coverages, interactions with only one or two adsorbed particles are relevant. Therefore, we simulated the Brownian motion of an incoming particle in the presence of, respectively, one and two adsorbed spheres on the surface. The center of the moving particle diffuses in the region shown in Fig. 1. Its trajectory was simulated by using the classical algorithm of Ermak and McCammon [12]: Given the position of the particle at time $t$, its displacement along the $i$ th coordinate axis during a small interval of time $\Delta t$ is calculated according to

$\Delta x_{i}=\sum_{j=1}^{3}\left(\frac{\partial D_{i j}}{\partial x_{j}}-\frac{D_{i j}}{k T} \frac{\partial U}{\partial x_{j}}\right) \Delta t+\Delta X_{i}, \quad i=1,2,3$

$\Delta X_{i}$ is a Gaussian random variable with zero mean and variance given by $\left\langle\Delta X_{i} \Delta X_{j}\right\rangle=2 D_{i j} \Delta t$. All the quantities are computed at the position of the particle at time
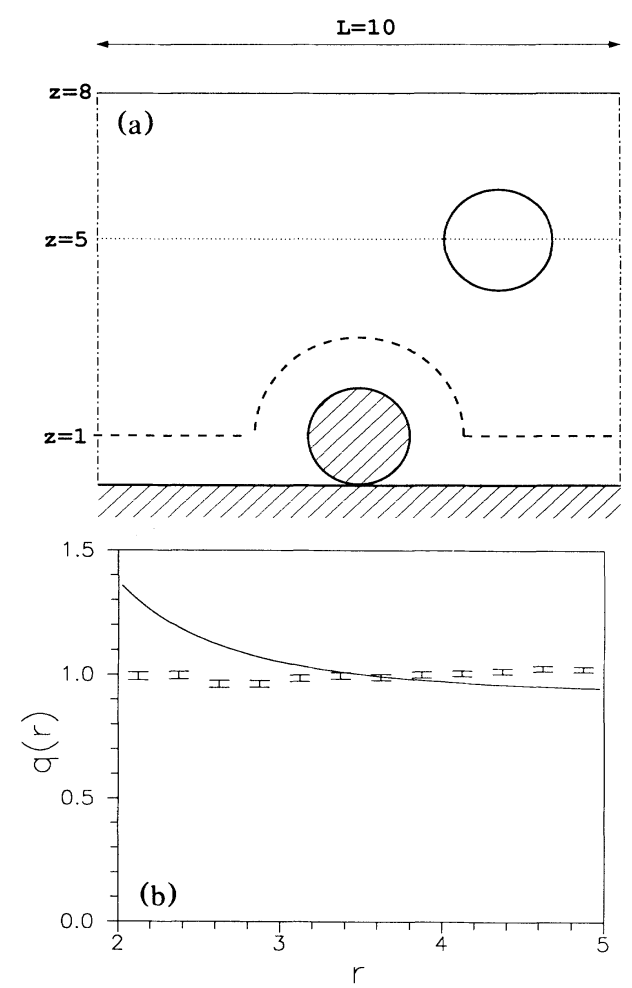

FIG. 1. (a) Geometry of the simulation. The region from which the center of the diffusing particle is excluded is delimited by the dashed line (exclusion surface). (b) Relative distribution of the points of adsorption of particles obtained by simulation of $10^{5}$ trajectories with $A_{\text {adh }}=0.4426$. The results of the simulation are compared to the distribution obtained when the particles diffuse in the absence of hydrodynamic interactions and van der Waals forces (DRSA, continuous line).

$t$. The time interval $\Delta t$ is taken so small that the mean magnitude of the displacement $\delta=|\Delta \mathbf{x}|$ is smaller than the characteristic length on which $D$ and $\partial U / \partial x$ change significantly. The value of $\delta$ in the bulk, $\delta_{0}$, fixes the spatial resolution of the simulation. It should be a small fraction of the radius of the particle, i.e., $\delta_{0} \ll a$. When the center of the particle approaches the exclusion surface (Fig. 1), the components of the diffusion tensor and the van der Waals potential depend strongly on the distance, $s$, to the surface which becomes the relevant distance. The step of integration is then modified to become of the order of $\delta=s \delta_{0} / a$. This process is stopped at some small, but finite distance, to avoid an infinite number of elementary displacements and thus an infinite computer time.

Fortunately, the behavior of the particles at sufficiently small separations can be obtained using the asymptotic behavior of the diffusion tensor $\left[D_{\perp} \sim s, D_{\|} \sim-(\ln s)^{-1}\right]$ and of the potential $U \sim s^{-1}$. Because we do not take attractive van der Waals forces between particles into account, the surfaces of the adsorbed spheres are perfectly reflecting boundaries. The surface of the exclusion sphere 
around a fixed particle is then an entrance boundary [13] that cannot be reached by the center of the diffusing particle. Spheres arriving at a small distance $\epsilon_{1} \ll a$ will then be reflected at some position on the spherical surface at a distance $2 \epsilon_{1}$ from the particle. Using the expressions of the potential and of the diffusion coefficients near the surface, the mean time $\tau$ for this diffusion process can be computed using Eq. (5.2.160) of Ref. [13]. The lateral displacement of the particle can then be estimated by $\left(\left\langle\Delta r_{\|}^{2}\right\rangle\right)^{1 / 2} \approx\left(4 D_{\|} \tau\right)^{1 / 2} \approx \sqrt{4 \epsilon_{1} a}$. It is taken smaller than the resolution $\delta_{0}$, and therefore one takes $\epsilon_{1}=\delta_{0}^{2} / 4 a(\ll a)$. Near the adsorbing surface, in contrast, the attractive van der Waals force prevents the particle from escaping; the adsorbing surface is then an exit boundary [13]. The simulation is stopped when the particle reaches a distance $\epsilon_{2}$ : A particle starting from a plane of height $\epsilon_{2}$ eventually reaches a surface at height $2 \epsilon_{2}$ with a predetermined small probability $p$. Using Eq. (5.2.189) of Ref. [13] one finds $\epsilon_{2} \approx a A_{\mathrm{adh}} /(-2 \ln 4 p)$ with $A_{\text {adh }}=A_{H} / 6 k T$. The mean lateral displacement during this step is $\epsilon_{2} \sqrt{2 / A_{\text {adh }}}$, which should be smaller than $\delta_{0}$.

Taking the radius of the particles $a$ as the unit of length, and the time $t_{0}=a^{2} / D(\infty)$ as the unit of time, the problem can be cast in dimensionless form. The only dimensionless parameter is the adhesion number $A_{\mathrm{adh}}$, measuring the relative strength of the van der Waals force with respect to the random force. If $A_{\text {adh }} \gg 1$ the trajectory of the particle becomes deterministic for $s \sim 1$, and a distribution similar to that obtained in ballistic deposition models has to be expected. If, on the contrary, $A_{\mathrm{adh}}<1$, the diffusive motion is dominant until the particle is very near to the surface; a homogeneous distribution is to be expected. For polystyrene particles in water at $300 \mathrm{~K}$ one has $A_{\mathrm{adh}}=0.4426$. In the following, all the quantities will be expressed in a dimensionless way.

For the case of one adsorbed particle the simulation procedure is as follows: One particle is permanently fixed at the center of a square of side 10 , with its center at height $h=1$. This square is surrounded by four vertical walls to which periodical boundary conditions are applied. The periodicity did not influence the results. The starting position of the center of the moving particles is chosen randomly on a horizontal plane at a height $h$ above the surface. Afterwards, the values of the diffusion matrix and the force corresponding to the position of the particle are computed as described previously. The displacement of the particle is then determined by using Eq. (2). It was verified on preliminary simulations that long-range hydrodynamic interactions do not modify significantly the random distribution of particles if $h \geq 5$. Thus, the starting height was fixed at $h=5$. Furthermore, to avoid particles escaping towards infinity, a sphere reaching the plane $h=8$ was rejected, and a new one restarted from a random position in the plane $h=5$. The mean displacement of the particle in each step is initially $\delta_{0}=0.1$. The value of $p$ was chosen to be $10^{-5}$. Therefore, $\epsilon_{1}=0.0025$ and $\epsilon_{2}=0.022$. Once the particle touches the adsorbing plane, without overlap with the fixed sphere, the coordinates $(x, y)$ of the contact point are recorded. The particle is then removed and a new one started randomly from $h=5$. This procedure is repeated until a chosen number of particles, $10^{5}$ in the present study, has reached the surface. At the end of the simulation stage, the surface is divided into small regions of size $d$. The ratio $q(r)$ of the density of contact points (recorded previously) at a distance $r$ from the fixed particle to the average density is plotted in Fig. 1, together with the corresponding result obtained ignoring hydrodynamic interactions and van der Waals forces (DRSA). It shows that the effect of the hydrodynamic interactions is to cancel out practically the nonuniformity introduced by diffusion. One recovers in this way a uniform distribution. This is precisely the basic hypothesis of the RSA that seems, therefore, to be a quite correct model to describe the structure of the adsorbed phase at least at low coverages.

To verify this conclusion for higher coverages, we performed also simulations in the presence of two adsorbed particles, fixed on a rectangular $15 \times 10$ surface, the distance between their centers being 5 [Fig. 2(a)]. Periodic boundary conditions were applied as previously. The re-

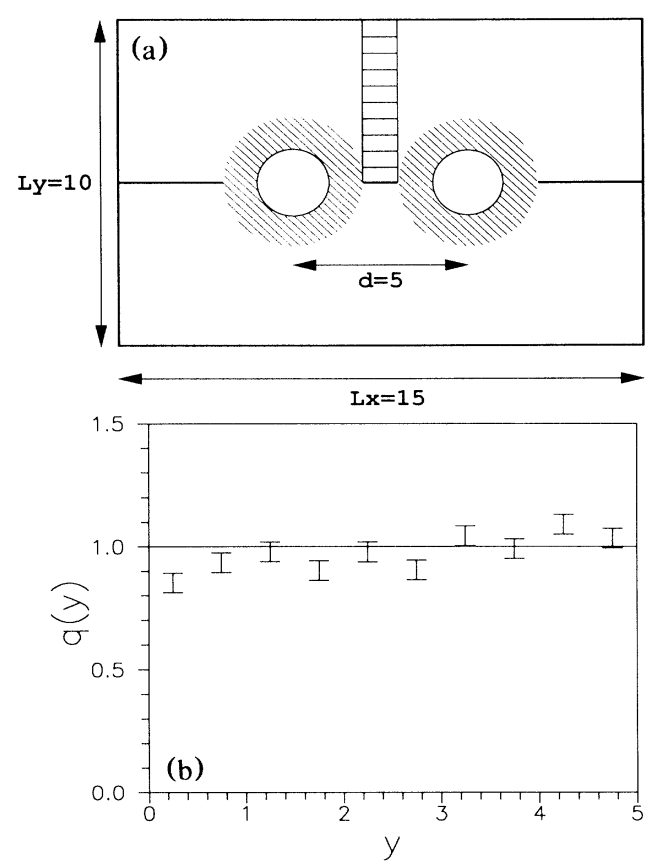

FIG. 2. (a) Geometry of the simulation with two spheres fixed on the surface. (b) Adhesion probability along the axis perpendicular to the line of centers of two spheres fixed on the surface. Each point corresponds to one of the rectangles in (a), $y=0$ being the center of the simulation area. 
mainder of the simulation procedure is identical to the one-sphere case. There is now a relatively small region between both spheres for the deposition of a third one, and the effects of the hydrodynamic interaction are expected to be large there. Figure 2(b) shows the probability density for the adhesion of a third particle along the axis normal to the line of centers of the fixed spheres. A small depletion (of the order of $10 \%$ ) seems to appear in the region nearest to the spheres. Away from that region, the distribution is uniform within statistical error. We can understand these results in the following way: The most important effect of the hydrodynamic interactions is to diminish the motion perpendicular to the surface in comparison to the lateral motion. Therefore the lateral diffusion homogenizes the distribution of particles. Thus, they reach the surface in a practically homogeneous way. The inclusion of repulsive electrostatic forces between the moving particle and the adsorbing surface would even strengthen this effect: If the particles meet a repulsive barrier or a secondary minimum before reaching the surface, they will diffuse for a long time parallel to the surface before being adsorbed.

The main goal of this study was to introduce accurately the hydrodynamic interactions during the adhesion process of spherical particles on a solid surface. We have treated here the case in which interparticle interactions other than hydrodynamic or hard core are neglected. In this case, the particles seem to adsorb almost randomly on the surface without correlation with previously adsorbed spheres. This implies that, despite the complexity of the adhesion phenomenon, RSA is a suitable model to describe accurately the particle distribution on the surface. This result is, at first sight, surprising and gives a new validity to the numerous RSA studies undertaken during the last years. Even though the results reported in this Letter can only lead to this conclusion for coverages lower than or of the order of $30 \%$ (for higher coverages three-particle interactions become important [14]), it can be assumed that it remains valid up to the jamming limit.
Indeed, for high coverages, only small regions in space remain accessible to the centers of new particles. Then, during the diffusion of the particles toward the surface, a randomization of the particle in the direction parallel to the surface is likely to occur. Further studies are now under way to include van der Waals and double layer forces between particles in the model.

This work has been partly supported by NATO Grant No. 890872. Two of us (J.B. and P.S.) acknowledge the C.E.C. for its financial support [Contract No. SC1915166 and Contract No. SC1-CT-91-0696 (TSTS), respectively]. We thank D. Bedeaux, G. Koper, and M. Rubí for helpful discussions.

[1] E. L. Hinrichsen, J. Feder, and T. Jøssang, J. Stat. Phys. 44, 793 (1986).

[2] B. Senger, J.-C. Voegel, P. Schaaf, A. Johner, A. Schmitt, and J. Talbot, Phys. Rev. A 44, 6926 (1991).

[3] G. Tarjus and P. Viot, Phys. Rev. Lett. 68, 2354 (1992).

[4] R. P. Feynman, The Feynman Lectures on Physics (Addison-Wesley, Reading, 1966).

[5] J. Happel and H. Brenner, Low Reynolds Number Hydrodynamics (Martinus Nijhoff, Dordrecht, 1986).

[6] T. van de Ven, Colloidal Hydrodynamics (Academic, London, 1989).

[7] H. C. Hamaker, Physica (Utrecht) 4, 1058 (1937).

[8] D. J. Jeffrey and Y. Onishi, J. Fluid Mech. 139, 261 (1984).

[9] G. Bossis, A. Meunier, and J. D. Sherwood, Phys. Fluids A 3, 1853 (1991).

[10] H. Brenner, Chem. Eng. Sci. 16, 242 (1961).

[11] A. J. Goldmann, R. G. Cox, and H. Brenner, Chem. Eng. Sci. 22, 637 (1967).

[12] D. L. Ermak and J. A. McCammon, J. Chem. Phys. 69, 1352 (1984).

[13] C. W. Gardiner, Handbook of Stochastic Methods (Springer, Berlin, 1990).

[14] P. Schaaf and J. Talbot, Phys. Rev. Lett. 62, 175 (1989). 